\title{
Reaching in Depth: Hand Position Dominates over Binocular Eye Position in the Rostral Superior Parietal Lobule
}

\author{
Stefano Ferraina, Emiliano Brunamonti, Maria Assunta Giusti, Stefania Costa, Aldo Genovesio, and Roberto Caminiti \\ Department of Physiology and Pharmacology, Sapienza University of Rome, 00185 Rome, Italy
}

\begin{abstract}
Neural activity was recorded in area PE (dorsorostral part of Brodmann's area 5) of the posterior parietal cortex while monkeys performed arm reaching toward memorized targets located at different distances from the body. For any given distance, arm movements were performed while the animal kept binocular eye fixation constant. Under these conditions, the activity of a large proportion (36\%) of neurons was modulated by reach distance during the memory period. By varying binocular eye position (vergence angle) and initial hand position, we found that the reaching-related activity of most neurons (61\%) was influenced by changing the starting position of the hand, whereas that of a smaller, although substantial, population (13\%) was influenced by changes of binocular eye position (i.e., by the angle of vergence). Furthermore, the modulation of the neural activity was better explained expressing the reach movement end-point, corresponding to the memorized target location, in terms of distance from the initial hand position, rather than from the body. These results suggest that the activity of neurons in area PE combines information about eye and hand position to encode target distance for reaching in depth predominantly in hand coordinates. This encoding mechanism is consistent with the position of PE in the functional gradient that characterizes the parieto-frontal network underlying reaching.
\end{abstract}

\section{Introduction}

The transformation of visual information into plans for action depends on the integrity of posterior parietal cortex (PPC). Patients with PPC lesions recognize objects, but not their spatial relationships (Critchley, 1953), especially in depth (Brain, 1941). Optic ataxia, from lesions centered on the superior parietal lobule (SPL) (for review, see Battaglia-Mayer and Caminiti, 2002; Caminiti et al., 2005), prevents accurate performance of hand reaches to visual targets. These patients exhibit a specific deficit in localizing visual targets with respect to their body (Holmes and Horrax, 1919; Rondot et al., 1977; Danckert et al., 2009) as well as in the integration of visual and proprioceptive information (Blangero et al., 2007, 2008; Khan et al., 2007) about hand position.

The parietal mechanisms of reaching have been studied extensively in a two-dimensional space (Kalaska et al., 1983; Ferraina et al., 1997, 2001; Batista et al., 1999; Battaglia-Mayer et al., 2000; Buneo et al., 2002). Thanks to these studies, different encoding mechanisms have been proposed (Cohen and Andersen, 2002; Battaglia-Mayer et al., 2003). So far, the parietal encoding of distance for reaching in three dimensions has been the subject of only few studies (Lacquaniti et al., 1995; Bhattacharyya et al., 2009), which, however, did not evaluate the respective influence

Received March 17, 2009; revised July 20, 2009; accepted Aug. 3, 2009.

This work was supported by Ministero Istruzione Università e Ricerca of Italy (PRIN 2004057380 and 2005051741 ; FIRB RBNE01SZB4_007), partially by Italian National Institute of Health Grant 530/F4/1, and by Sapienza University of Rome Grant C26G031031.

Correspondence should be addressed to Prof. Stefano Ferraina, Department of Physiology and Pharmacology, Sapienza University of Rome, Piazzale Aldo Mor0 5, 00185 Rome, Italy. E-mail: stefano.ferraina@uniroma1.it. DOI:10.1523/JNEUROSCI.1305-09.2009

Copyright $\odot 2009$ Society for Neuroscience ～0270-6474/09/2911461-10\$15.00/0 of eye and hand positional signals in the composition of commands for arm movements.

The localization of a visual target in three-dimensional (3D) space depends on the combination of binocular signals, such as retinal disparity, with fixation distance information (Pouget and Sejnowski, 1994). The latter depends on a variety of signals, such as the binocular eye position (vergence angle), accommodation, and vertical disparity, although vergence seems to be the most important one (von Hofsten, 1976; Foley, 1980). Vergence cues are particularly relevant within reaching distance, as most of the vergence range is used for fixation distances that are approximately equal to the arm length (Viguier et al., 2001). Interestingly, in infants, reaching develops at the same time as binocular control (von Hofsten, 1977).

Area PE (Pandya and Seltzer, 1982) in the SPL is reciprocally linked to M1 (Johnson et al., 1996). It receives limb position information from SI (Jones et al., 1978) and visuomotor signals from PEc (Marconi et al., 2001). Its role in visually guided reaching is supported by different neurophysiological studies (for review, see Battaglia-Mayer et al., 2003). However, it is unknown whether or not PE neurons encode the location of targets in depth and whether their neural activity is modulated by information such as binocular eye position and initial hand position.

The results of the present experiment show that PE's neural activity is monotonically tuned to hand reaching toward targets located at different distances from the body. Furthermore, this reaching-related activity is strongly modulated by the hand's position, and more weakly by the binocular eye position. Finally, the memorized target distance is encoded relative to the initial hand position rather than to the body, 
Table 1. Relative distance from the monkey body of targets, FPs, and buttons used as the starting position for each monkey

\begin{tabular}{lll}
\hline & Monkey UM & Monkey IS \\
\hline Target 1 & 130 & 100 \\
Target 2 & 180 & 160 \\
Target 3 & 230 & 220 \\
Target 4 & 280 & \\
Target 5 & 330 & $100\left(20^{\circ} \mathrm{VA}\right)$ \\
Fixation 1 & $200\left(10^{\circ} \mathrm{VA}\right)$ & $140\left(15^{\circ} \mathrm{VA}\right)$ \\
Fixation 2 & $250\left(8^{\circ} \mathrm{VA}\right)$ & $195\left(10^{\circ} \mathrm{VA}\right)$ \\
Fixation 3 & $312\left(6^{\circ} \mathrm{VA}\right)$ & \\
Starting position 1 & 150 & \\
Starting position 2 & 200 & \\
Starting position 3 & 250 & \\
\hline
\end{tabular}

Values are indicated in millimeters. Vergence angles (VA) for fixation distances are indicated in degrees.

supporting the existence of a hand-centered reference frame for reaching in depth in the rostral part of the SPL.

\section{Materials and Methods}

Animals, surgery, and experimental setup. Two rhesus monkeys (Macaca mulatta; monkeys UM and IS; body weight: UM, $7.8 \mathrm{~kg}$; IS, $4.8 \mathrm{~kg}$ ) were studied using general procedures similar to those described previously (Genovesio and Ferraina, 2004), and further outlined here. Animal care, housing, and surgical procedures were in conformity with the European (Directive 86/609/ECC) and Italian (D.L. 116/92) laws on the use of nonhuman primates in scientific research.

Animals were preanesthetized with ketamine $(10 \mathrm{mg} / \mathrm{kg}$, i.m. $)$ and anesthetized with isofluoran (Abbott Laboratories) through a constant flux of isofluoran/air mixture (1-3\%, to effect). Under such conditions, a recording cylinder was implanted at known stereotaxic coordinates (monkey UM, P3-L14; monkey IS, P3-L10) to allow extracellular recordings of single-unit activity from area PE (see Fig. 1A). Binocular scleral search coils and a head post were also implanted aseptically during the same surgical session.

During the experiments, the monkeys sat in a primate chair with the head restrained and faced two robotic arms (CRS Robotics) used to position light-emitting diodes (LEDs) in depth (Genovesio and Ferraina, 2004). One of the two robots was used to position the fixation point (FP), which was aligned to the body's midline at eye level. Three different fixation distances were used (see Fig. 1B, F1-F3), corresponding to different values of the vergence angle (Table 1), as obtained by taking into account the interocular distance of the monkeys (monkey UM, $35 \mathrm{~mm}$; monkey IS, $36 \mathrm{~mm}$ ). To prevent the FP from illuminating the robot arms, the light from the corresponding LED was presented by the tip (diameter, $0.5 \mathrm{~mm}$ ) of an optic fiber. The second robot was used to position the target $(\mathrm{T})$ for the required arm movement. Targets were placed at constant elevation and aligned, both horizontally and vertically, to the shoulder. The target was only briefly presented by the illumination of an LED attached to the tip of the robot arm that removed the physical target after its presentation. To position targets at a comfortable reaching distance, different target location arrangements were used for the two monkeys, based on the arm length. In monkey UM, we used five targets (T1-T5; 5 cm steps) (see Fig. $1 B$ ), at depths of $13-33 \mathrm{~cm}$ from the shoulder. In monkey IS, we used three targets (T1-T3; $6 \mathrm{~cm}$ steps), at depths of 10-22 $\mathrm{cm}$. For both animals, three push-buttons were attached to the chair on the animal's side (see Fig. $1 B, \mathrm{H} 1-\mathrm{H} 3$ ), $26 \mathrm{~cm}$ below the shoulder and at different distances from it $(15,20,25 \mathrm{~cm})$. They were used as different starting positions for the hand. Push-buttons were independently illuminated by an LED only at the onset of each trial, to help monkeys to place the hand at the required initial position. All tasks were performed in total darkness, and the room was illuminated during the intertrial interval to avoid dark adaptation.

Arm position and trajectory were monitored by using an optoelectronic motion capture system (Optotrack 3020 real-time system; Northern Digital), at a $100 \mathrm{~Hz}$ sampling rate. To record the hand trajectory and control the movement end-point (see Fig. $1 B$ ), three optic markers were placed on a bracelet mounted to the wrist of the monkeys. The position of the fingers used to compute the reach end-point to the memorized target locations was calculated from the position of the three optic markers. The Optotrack system was also used to calibrate the robot's work space in a reference system centered on the monkey's head, at the midpoint level of the interocular distance (i.e., the cyclopean eye). Optic markers were attached to the robot's joints and used to control the position of both the FP and the target. The shoulder position was measured during the calibration procedure. This was repeated at the beginning of each recording session. Each value obtained from the Optotrack system was then recorded during the experiment and referred to the origin of the work space.

All neural recordings were made in the hemisphere contralateral to the performing arm. Single-unit activity was isolated from the extracellular recorded activity using a dual time-amplitude window discriminator (BAK Electronics). The electrodes used were glass-coated tungsten-platinum fibers $(0.8-2 \mathrm{M} \Omega$ impedance at $1 \mathrm{kHz})$.

Behavioral task. Monkeys were trained in a memory-delayed task in which reaches were made to extrafoveal targets presented in 3D space. To avoid overreaching strategies, hand movements were made in the dark toward memorized targets, therefore in the absence of any physical object to touch. Each trial started when the monkey placed its hand on the lighted push-button used as the starting hand position (see Fig. 1C). Then, the LED on the push button was turned off, and the FP was turned on. The monkey was required to fixate binocularly the FP for a variable control time (500-800 ms), at the end of which the peripheral LED was briefly $(300 \mathrm{~ms})$ flashed signaling the target position, and the robot arm holding the target was removed. After a variable (500-800 ms) memory delay period, the FP changed color (green), as a go-signal for reaching to the memorized target location (movement time; upper limit, $1000 \mathrm{~ms}$ ), while maintaining fixation stable. The reward was delivered after a variable (200-500 ms) holding time of the hand at the location of the memorized target.

The error rate for each movement end-point was explored. In both animals, there was evidence of overestimation of the closer target and underestimation of the farther target location. However, a suitable estimate of movement end-point errors was difficult to achieve. The main reason was that the hand's position was derived from the three wired optic markers arranged as bracelet at the level of the wrist. This marker's location in the distal part of the arm was affected by a systematic error in the computation of the angle between the hand and the wrist. However, we were able to compensate for this error and control the behavior efficiently (i.e., to obtain for each monkey separate trajectories/end-point replicas for each of the memorized targets) by introducing on-line corrections to the experimentally measured values.

Monkeys performed two versions of the reaching task. In the first version, both target and fixation distance were changed between trials in an intermingled design, whereas the hand starting position remained fixed. In the second version, both target and the initial hand position were changed between trials, whereas fixation distance remained constant. We required a minimum of five trials for each condition.

For both monkeys, eye signal calibration was obtained by requiring the monkeys to fixate sequentially five LEDs, one central and the others positioned to form an angle of -10 and $+10^{\circ}$, both in the vertical and in the horizontal axis of the isovergence surface corresponding to $10^{\circ}$ of vergence angle (see also Genovesio and Ferraina, 2004). During calibration, the central LED was aligned with the eye that was being calibrated, while the other eye was kept covered. The same calibration procedure was then repeated for the other eye. During the task, monkeys were required to maintain binocular fixation on the FP within a $1^{\circ}$ (radius) spherical window until the end of the trial. The control was maintained separate for each eye. The spherical tolerance window for the hand movement end-point was set at $3 \mathrm{~cm}$ (diameter). The accuracy of the vergence angle from trial to trial was evaluated by off-line inspection. More than $80 \%$ of the trials resulted in a vergence error of $<0.3^{\circ}$. All trials with vergence errors $>0.5^{\circ}$ were excluded from the analysis. The entire dataset was discarded when, after off-line inspection, less than three trials remained available for each combination of target, fixation distance, and initial hand position. 
A

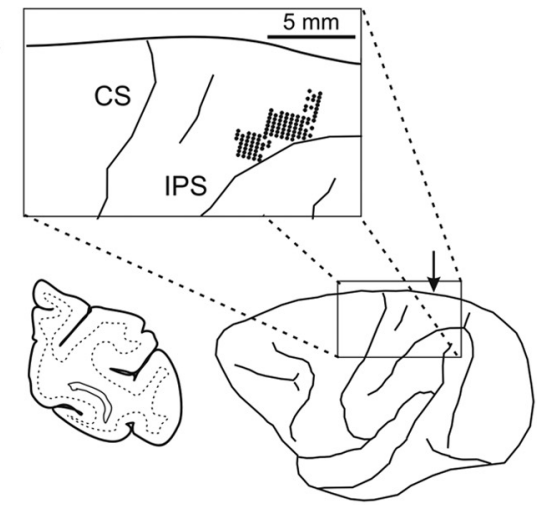

B

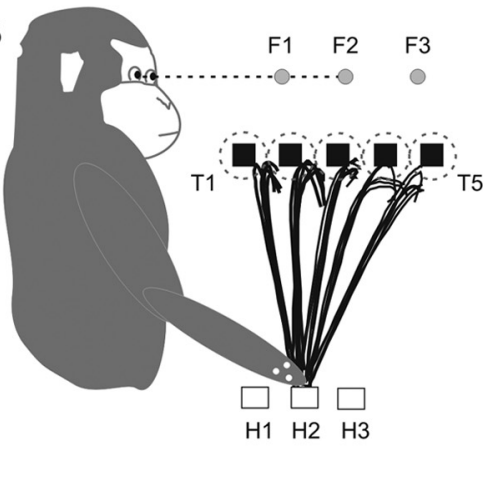

C
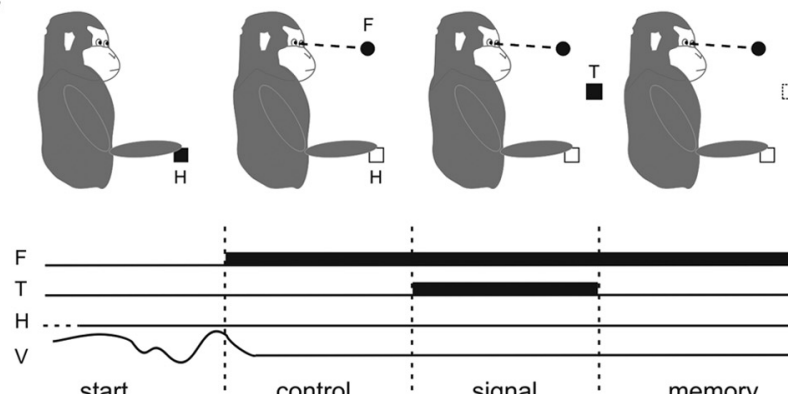

:

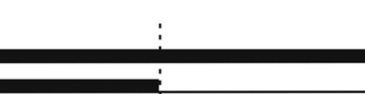

Figure 1. Schematics of the recording locations, work-space arrangement, and task. $\boldsymbol{A}$, Lateral view of the brain from one monkey (UM), illustrating the region of recording, as reconstructed after the histological procedures. Electrode entry points are shown as large black dots in the inset. The arrow in the brain figurine indicates the level of the coronal section shown in the left portion of the panel. IPS, Intraparietal sulcus; CS, central sulcus. $\boldsymbol{B}$, Illustration of a representative recording session. Monkeys performed reaching movements toward five different targets, located at five different distances from the body, starting from one of three hand positions. During each trial, the monkeys fixated binocularly at one of three FP locations. The trajectory and the final hand position were detected by reading optoelectric signals generated by three markers (white dots) attached to the monkey's wrist (see Materials and Methods for details). The reaching trajectories from the position $\mathrm{H} 2$ toward the five targets are represented by different groups of partially overlapped trajectories. Each line within the same group represents a trial ( 5 total) directed to a common memorized target. Dashed circles indicate the tolerance window for the hand movement end-points. $\boldsymbol{C}$, The binocular eye position $(\mathrm{V})$, the hand position $(\mathrm{H})$, and the time of presentation of the visual stimuli ( $\mathrm{F}$, fixation point; $\mathrm{T}$, target) are shown as a function of time. All trials began with an LED illuminating the push button used as the starting position for hand movement. As soon as the monkey touched the button, a central stimulus (F) was presented by one of the two robot arms. Monkeys were required to fixate and maintain fixation until the end of the trial. After a variable holding time (500-800 ms), a target appeared at one of five (3 in monkey IS) locations in depth and remained on for $300 \mathrm{~ms}$ (signal period). Then, the second robot arm moved, removing the target at the end of the signal epoch. After a variable 500-800 ms memory delay period, F changed color (green), as a go-signal for the monkey to reach to the memorized target location (response).

Data analysis. The rasters of neuronal discharges were aligned to specific behavioral events. Raw spike counts and spike density functions were used to measure the neural activity during different epochs of the task. "Control activity" was defined as the mean discharge rate from -500 to $-200 \mathrm{~ms}$ before target presentation; "signal activity" was the mean discharge rate of a $200 \mathrm{~ms}$ window starting $70 \mathrm{~ms}$ after target presentation; "memory activity" was defined as the mean discharge rate of the last $300 \mathrm{~ms}$ of the memory delay epoch, ending when the FP changed color; "movement activity" was the mean discharge rate of the $200 \mathrm{~ms}$ window centered on the onset of hand movement. To generate spike density functions, a Gaussian pulse $(\sigma=15 \mathrm{~ms})$ was substituted to each spike, and all Gaussians were summed to produce a continuous function in time.

Significant modulations of neuronal activity in different task epochs, relative to the control activity, were assessed by an ANOVA and the Dunnett's post hoc test. Significant effects on neural activity of target distance, fixation distance, and/or initial hand position were evaluated through both a three-way ANOVA and a multiple linear regression model. The three-way ANOVA used the control activity as the covariate. The significance level for all statistical tests was set at 0.05 . To describe the relationship between average activity and target distance, the cells with significant effect for target distance were further analyzed through an
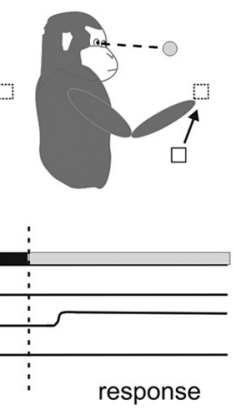

ANOVA with orthogonal polynomial contrasts. The quadratic polynomial tested whether quadratic relationships exceeded linear ones; the cubic polynomial tested whether cubic fits exceeded quadratic ones.

The hand's distance error for each target (i.e., the difference in depth between movement end-point and initial position of the hand) was computed off-line using kinematics data obtained through the Optotrack system. Since no physical target was presented, the final hand position (movement end-point) was derived from the descending portion of the peakvelocity curve, using as threshold the time when the hand's velocity reached the $10 \%$ of the peak velocity value.

Histological procedures. At the end of the experimental procedures, the first animal (monkey UM) was anesthetized (sodium pentobarbital; $50 \mathrm{mg} / \mathrm{kg}$, i.v.) and perfused with $0.9 \%$ saline, followed by $4 \%$ paraformaldehyde in $0.1 \mathrm{M}$ phosphate buffer, $\mathrm{pH} 7.2$; the dura was opened, and four pins were inserted at known chamber coordinates to delimit the area of recording. After removal, the brain was postfixed in the same fixative and placed in a solution of $30 \%$ buffered sucrose, until it sank. The brain blocks were cut in the coronal plane. Sections (40 $\mu \mathrm{m}$ thick) were stained with thionin $(0.025 \%)$ for cytoarchitectonic analysis. The borders of different parietal areas were drawn on the histological material, by using the criteria defined in the literature (Pandya and Seltzer, 1982). The second monkey is currently involved in a different experimental protocol; therefore, the histological material is not available yet. However, the consistency of the results across animals suggests that, in both of them, the area of recording was primarily similar.

\section{Results}

We recorded the activity of 319 neurons in area PE of the SPL. Recording depth was confined within $2500 \mu \mathrm{m}$. The location of the neural recording was confirmed by visual inspections of the anatomical landmarks, such as the central sulcus and the intraparietal sulcus, as well as (on monkey UM) by the cytoarchitectonic analysis of the Nissl-counterstained material (Fig. 1A). Out of our initial database of neurons, 247 of 319 (77.4\%) were reaching-related, since their discharge rate in the memory and/or movement epoch was significantly different than that observed in the control time (ANOVA, $p<0.05$, Dunnett's post hoc).

\section{Reaching-related neurons in area $\mathrm{PE}$ are modulated by the distance of the target from the body}

We studied 207 reaching-related neurons (207 of 247; 83.8\%) while the animals made arm movements to memorized targets located at different distances from the body, starting from the intermediate hand position (H2) and maintaining binocular eye fixation to the intermediate fixation distance (F2). Both monkeys performed the task in a similar manner. Their average reaction time was $255 \mathrm{~ms}( \pm 66 \mathrm{SD})$, and their average movement time was $324 \mathrm{~ms}$ ( $\pm 64 \mathrm{SD})$. Figure 2 shows one representative parietal reaching neuron, tuned for target distance. The neural activity increased with target distance mostly during movement preparation (memory epoch) (Fig. $2 \mathrm{~A}$, gray area). 
The activity of 75 of 207 (36.2\%) reaching-related neurons was modulated by target distance (ANOVA, $p<0.05$ ) in the memory epoch, whereas that of 129 of $207(62.3 \%)$ neurons was modulated in both the memory epoch and in at least one of the other periods (signal or movement). The activity of 50 of 207 (24.1\%) neurons was modulated in both the memory and movement epochs. Only 13 of 207 (6.3\%) neurons were sensitive to target distance uniquely during the presentation of the visual signal about target location. The polynomial contrast analysis showed that 60 of $75(80 \%)$ of distance-modulated neurons displayed a significant $(p<0.05)$ linear trend between target distance and neural activity, and no relationships with quadratic or cubic models; $17 \%$ ( 13 of 75 ) of the neurons tested had a significant quadratic relationship, but not a cubic one (with the maximum never located at intermediate distance); the activity of only two neurons was better explained by a cubic model. In conclusion, approximately two-thirds of the PE neurons, when tested during reaching in depth, displayed a significant modulation for the target distance.

The next session will illustrate the results concerning the activity of reaching-related neurons studied when varying the binocular eye position in depth and the initial hand position. Since both conceptual approaches and experimental evidences suggest that the visuomotor transformation underlying reaching can be better studied in the memory epoch (Andersen et al., 1990, 1997), we will focus our report on the results obtained during this period. We obtained similar results during the signal and movement epochs; therefore, these results will be mostly presented in the tables.

\section{Influence of eye and hand position signals on parietal reaching-related activity}

To identify in area PE the reference frame used for reaching in depth, we examined the influence of binocular eye position and arm position signals on the activity of reaching-related neurons. The neural activities obtained when monkeys reached toward an identical array of target locations in space was tested under two conditions. In the first condition, arm movements were made while maintaining binocular eye position at three different fixation distances. In this condition, the monkey's initial hand position was fixed on the central button. In the second condition, reaching movements were made while maintaining binocular eye position at a single fixation distance, and the monkey's hand started from one of three initial positions. By using these two different conditions, we have examined the reaching-related activity in eye- or hand-centered coordinates.

We limited our analysis only to neurons (142) endowed with a complete dataset. Figure 3 shows a neuron studied in both task conditions. The reaching-related activity of this neuron was modulated by the target distance from the body and, at the same time, by both hand and eye position. The influence of the hand position was particularly prominent (Fig. $3 A, C, E$ ) compared to the rather modest modulation exerted by the binocular eye posi- tion (Fig. 3B-D). At the population level, we performed a threeway ANOVA, with target distance, fixation distance, and initial hand position as factors. The control time was used as the covariate. In the memory epoch, we found that the activity of 87 of 142 $(61.3 \%)$ neurons was influenced by the initial hand position, whereas the activity of only 18 of $142(12.7 \%)$ neurons was influenced by changes of the fixation distance. When considering the neurons modulated by target distance ( 66 of $142 ; 46.5 \%$ ), only 12 of $142(8.4 \%)$ were not influenced by either hand or binocular eye positions, suggesting that the reaching target was encoded in egocentric coordinates, either shoulder or head centered. Figure 4 and Table 2 show the results of the three-way ANOVA for all epochs. Although we observed an increase of the number of neurons modulated by each factor (Fig. 4) through the trial, their respective contribution did not change between epochs, indicating that there was not a transition between reference frames along task trials.

In conclusion, most reaching-related neurons in area PE were influenced by the initial hand position and by target distance, whereas only a few of them were influenced by fixation distance. Furthermore, the influence on the neural activity of the different factors (i.e., target distance, fixation distance, and hand position) increased through the trial from the target presentation to motor execution.

Influence of motor error on parietal reaching-related activity As described in the previous paragraph, the three-way ANOVA showed that the activity of PE's reaching neurons was influenced predominantly by two main signals, target location in depth and initial hand position. To further address this influence, we used data derived from the kinematics control. These data served to compute, on a trial-by-trial basis, the hand movement end-point (E) and, for each value of E, three relative distances (Fig. 5 top) (see also Materials and Methods for details), such as the distance 


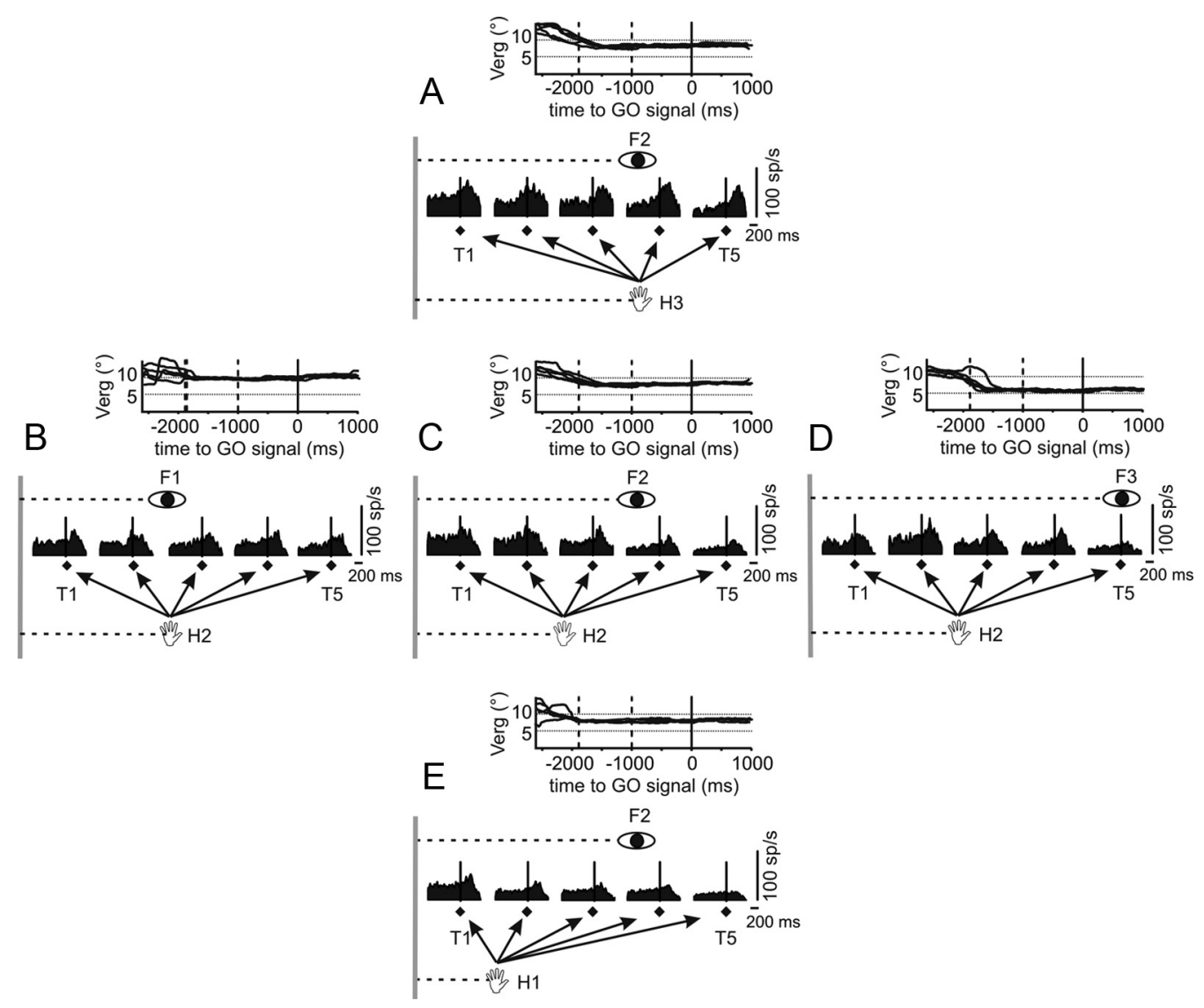

Figure 3. Influence of hand and eye positions on the modulation of a reaching neuron tuned for target distance. Spike density functions (SDFs) of reaching activities grouped for different targets (T1-T5; black diamonds) and aligned to the go signal (black vertical line) are shown. A, C, E, Data from a fixed eye position (F2) and different hand starting position (H1-H3). B-D, Data from a fixed hand starting position (H2) and different binocular eye positions (F1-F3). Calibration bars (time and discharge rate) for SDFs are indicated for each group. Gray vertical lines represent the origin of the work space, and horizontal dotted lines represents the distance of binocular eye position and hand position from the origin. Binocular eye position is shown, as function of time, for each groups of trials. Plots are aligned to the go signal (continuous vertical line) and displayed until the end of the trial. The vertical dotted lines in the vergence (Verg) plot indicate, from left to right, the average time of fixation onset and target presentation.

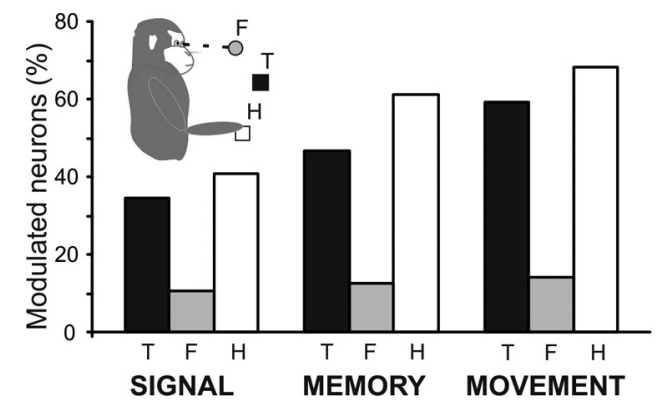

Figure 4. Results of the three-way ANOVA. Proportions of neurons modulated by target distance $(T)$, fixation distance $(F)$, and initial hand position $(H)$ during the different epochs analyzed (signal, memory, movement) are shown. The main and interactive effects of these factors are provided in Table 2.

from the FP (dFE), from the body (dBE), and from the initial hand position (dHE; i.e., the component in depth of the motor error). Then, we used these values to perform a multiple linear regression analysis, with neural activity as the dependent variable and the three distances as the independent factors. The regression analysis was used to determine which of the factors accounted for the variance of the dependent variable.

The preliminary step of the analysis was the control of the unacceptably high level of intercorrelation among the independent factors. When factors are more highly correlated with each
Table 2. Results of three-way ANOVA

\begin{tabular}{lclc}
\hline & Signal & Memory & Movement \\
\hline Main effect T & $38(26.8 \%)$ & $48(33.8 \%)$ & $70(49.3 \%)$ \\
Main effect E & $10(7.0 \%)$ & $13(9.1 \%)$ & $16(11.3 \%)$ \\
Main effect H & $50(35.2 \%)$ & $79(55.6 \%)$ & $94(66.2 \%)$ \\
Interactive effect T $\times$ E & $6(4.2 \%)$ & $8(5.6 \%)$ & $4(2.8 \%)$ \\
Interactive effect T $\times$ H & $16(11.3 \%)$ & $35(24.6 \%)$ & $43(30.3 \%)$ \\
Both T and interactive & $49(34.5 \%)$ & $66(46.5 \%)$ & $84(59.1 \%)$ \\
Both E and interactive & $15(10.6 \%)$ & $18(12.7 \%)$ & $20(14.1 \%)$ \\
Both H and Interactive & $58(40.8 \%)$ & $87(61.3 \%)$ & $97(68.3 \%)$ \\
\hline
\end{tabular}

Numbers and percentages of neurons with significant $(p<0.05)$ modulation for each factor (T, target distance; $\mathrm{E}$, binocular eye position; $\mathrm{H}$, initial hand position) considered and for the different epochs investigated are shown.

other than they are with the dependent variable, their copresence in the model must be reconsidered. We used the varianceinflaction factor (VIF) collinearity test and a threshold of VIF = 10 (Myers, 1986) as the limit for accepting the intercorrelation. Data related to all neurons of our sample were far below the threshold value adopted.

We found that in the memory epoch, 91 of $142(64.1 \%)$ neurons displayed a significant $(p<0.05)$ relationship with at least one factor. The activity of the majority of neurons was related either to $\mathrm{dBE}$ ( 72 of $91 ; 79.1 \%$ ) or $\mathrm{dHE}$ ( 80 of $91 ; 87.9 \%$ ). Only a minority of neurons was influenced by dFE (13 of 91; 14.3\%). Neural modulation was often explained by changes in more than one factor; in most instances by changes of both $\mathrm{dBE}$ and $\mathrm{dHE}(65$ 
of $91 ; 71.4 \%)$. Additional results from this analysis are provided in Figure 5 and Table 3. In particular, Figure 5 shows the number of neurons with a significant linear coefficient $(\beta)$ for each of the factors considered. Furthermore, Figure 6 contrasts, for each epoch, the partial correlation coefficients of neurons whose activity was significantly related to both $\mathrm{dBE}$ and dHE (overlapping regions in the Venn's diagram plots in Fig. 5). The partial coefficient, as part of the multiple linear regression analysis, measures the strength of the linear relationship between each factor and the dependent variable, controlling for the effect of the other factors (Dickey et al., 1998). Scatter plots show that during the visual signal epoch, the neural activity had a higher correlation with dHE in 36 of $51(70.6 \%)$ neurons. This proportion of neurons increased to $72.3 \%$ (47 of 65 neurons) in the memory epoch and to $77 \%$ (47 of 61 neurons) in the movement epoch.

Overall, the results from the three epochs support a prevailing influence of dHE over dBE. In fact, when considering all the neurons whose activity was explained preferentially by a single factor (merging the neurons with a main factor from the results in Table 3 with those with a greater partial correlation in Fig. 6), approximately two-thirds of them displayed a modulation of activity better explained by $\mathrm{dHE}$ (Fig. 7), whereas the activity of less than one-third of them was better explained by dBE.

Finally, when considering the sign of the relationship (partial correlation coefficients) of the neural activity with the distance between initial and final hand positions (dHE), we found a slighter prevalence of neurons preferring positive values over negative ones of the motor error signal, suggesting a preference for reaches away rather than toward the body. Neurons with a preference for positive values were $63.5 \%$ (40 of 63) in the signal epoch, $56.9 \%$ ( 41 of 72 ) in the memory epoch, and 59.4\% (44 of $74)$ in the movement epoch.

In conclusion, we found only a moderate influence on neural activity of the distance of the target from the fixation plane, whereas most of the neurons tested were influenced by changes in the distance between target and initial hand position. This last factor emerged as the dominant one in explaining the modulation of the neural activity in area PE.

\section{Discussion}

Our results show that the activity of reaching neurons in the rostral part of the SPL is tuned to reach distance. Furthermore, reach neurons combine binocular eye position information about fixation distance (i.e., the angle of vergence) with hand position signals. However, hand position influences reachingrelated activity more than fixation distance. Our experiment was designed to investigate a particular form of reaching (i.e., hand movement toward nonfoveated targets). Future studies need to explore the role of eye and hand signals on PE's neuronal modulation during reaching toward foveated targets in depth.

Most of the neurophysiological studies that so far have investigated reaching in 3D space (Schwartz et al., 1988; Caminiti et al.,
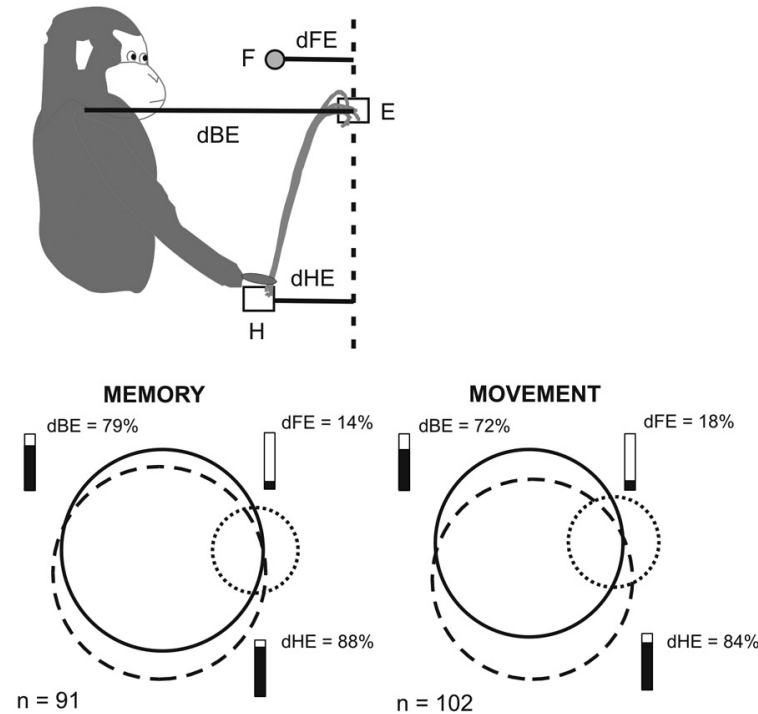

Figure 5. Results of the multiple linear regression analysis. Top, Schematic of the distances used for multiple linear regression Venn's diagram showing the relative proportion of the significant $(p<0.05)$ linear coefficients $\beta$ for each of the factors $d B E$, $d F E$ actor's relative frequency Overlapping regions indicate proportion of neurons influenced by the factors represented by the Additional details are provided in Table 3.

Table 3. Results of the multiple linear regression

\begin{tabular}{lccc}
\hline & Signal $(n=89)$ & Memory $(n=91)$ & Movement $(n=102)$ \\
\hline $\mathrm{dHE}$ & $18(20.4 \%)$ & $13(14.3 \%)$ & $20(19.6 \%)$ \\
$\mathrm{dBE}$ & $10(11.4 \%)$ & $5(5.5 \%)$ & $9(8.8 \%)$ \\
$\mathrm{dFE}$ & $6(6.8 \%)$ & $4(4.4 \%)$ & $3(2.9 \%)$ \\
$\mathrm{dHE}$ and dBE & $47(53.4 \%)$ & $60(65.9 \%)$ & $55(53.9 \%)$ \\
$\mathrm{dHE}$ and dFE & $2(2.3 \%)$ & $2(2.2 \%)$ & $5(4.9 \%)$ \\
$\mathrm{dBE}$ and dFE & $2(2.3 \%)$ & $2(2.2 \%)$ & $4(3.9 \%)$ \\
$\mathrm{dBE}, \mathrm{dFE}$, and dHE & $4(4.5 \%)$ & $5(5.5 \%)$ & $6(5.9 \%)$ \\
\hline
\end{tabular}

Numbers and percentages of neurons with significant $(p<0.05) \beta$ in all the epochs for each factor considered (dHE, dBE, dFE).

1990, 1991; Ferraina and Bianchi 1994; Lacquaniti et al., 1995; Johnson et al., 1996; Taira et al., 1996; Fattori et al., 2005, 2009) either explored only two depth planes or did not control for fixation distance. The importance of target distance coding has been explored recently by Caggiano et al. (2009) in mirror neurons studied in the ventral premotor cortex. These authors have shown that these neurons are tuned for target location relative to the observer's body. In the parietal lobe, evidence of distance coding is available from the study by Lacquaniti et al. (1995), showing that neurons in the SPL encode reaching in a bodycentered frame whose coordinates define the horizontal position (azimuth), elevation, and distance of the hand from the shoulder. In the present study, we only controlled for target distance. Target elevation and azimuth were kept constant; therefore, other studies are necessary to better explore their role. A reaching distance modulation of neural activity, as well as an influence of vergence has been reported recently in the parietal reach region (PRR) (Bhattacharyya et al., 2009).

\section{Vergence angle modulation of reaching-related activities for targets in depth}

The angle of vergence modulates the activity of a subpopulation of reaching-related neurons in area PE. The source of this binoc- 
SIGNAL

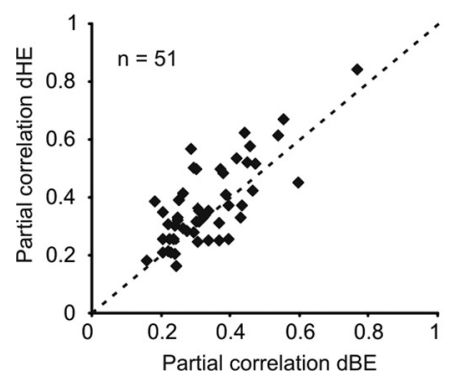

MEMORY

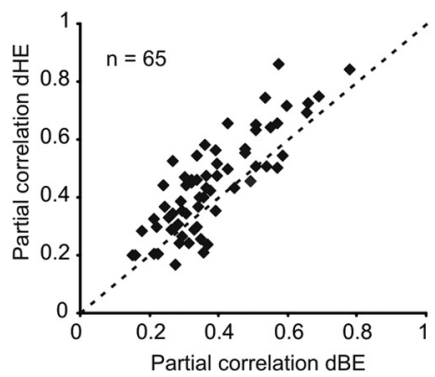

MOVEMENT

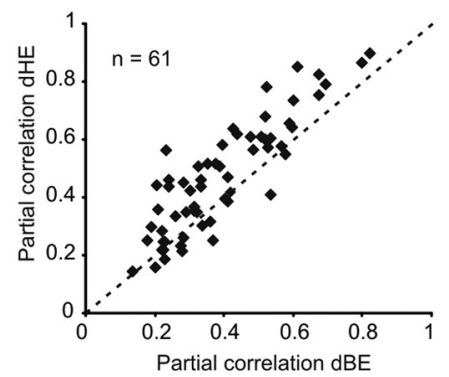

Figure 6. Contribution of the distance of the movement end-point (E) from either the body (dBE) or the initial hand position (dHE) to neural activity modulation. Scatter plots of partial correlation values for the hand-centered (dHE) and body-centered (dBE) relative distances of movement end-points. Only neurons with a significant correlation $(p<0.05)$ between the neural activity and each factor are shown. The dashed line indicates the equality line. Other conventions and symbols are as in Figure 5.

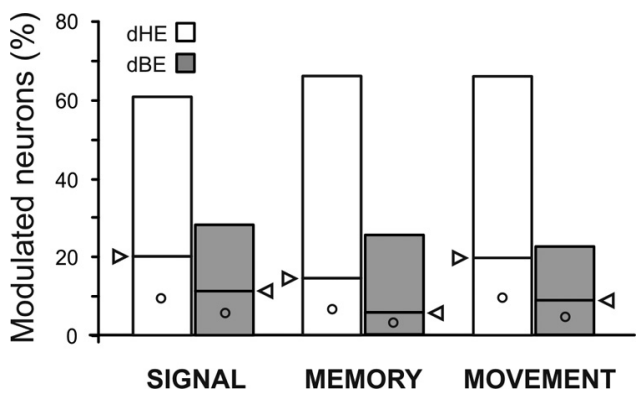

Figure 7. Proportion of neurons correlated to the distance of the movement end-point (E) either from the body (dBE) or from the initial hand position (dHE). Values represent, for each epoch, the sum of the neurons (bottom part of each column, marked by the symbol $O$ ) exclusively explained by a single factor (main effect in Table 3) and of those (top part of each column) explained prevailing by one of the two factors as resulting from the contrast (Fig. 6) obtained, using a winner-take-all rule, for those neurons with significant correlation $(p<0.05)$ for both factors. The white arrows indicate, for each column, the boundaries between the two classes of neurons. The prevailing effect of $\mathrm{dHE}$ is also evident considering only the main effect.

ular eye-position signal could be area PEc (Marconi et al., 2001), whose neural activity is modulated by eye-position information (Battaglia-Mayer et al., 2001; Ferraina et al., 2001; Raffi et al., 2008). In contrast, eye position signals modulate neural activity in area 3a (Zhang et al., 2008), the cortical field of projection of muscle spindle afferents (Oscarsson and Rosen, 1963), which is directly connected to area 5 (Darian-Smith et al., 1993). Last, a possible source of eye-position information is the central thalamus (Schlag-Rey and Schlag, 1984; Tanaka, 2007).

Previous studies have shown that the vergence angle modulates the activity of PPC neurons in both humans (Hasebe et al., 1999; Kapoula et al., 2001) and monkeys (Sakata et al., 1985; Gnadt, 1992; Genovesio and Ferraina 2004; Bhattacharyya et al., 2009). Fixation distance modulation of visual-related activity has been found in V1 (Trotter et al., 1996; Gonzalez and Perez, 1998), V2, V4 (Dobbins et al., 1998; Rosenbluth and Allman, 2002), and MST (Roy et al., 1992). In most instances, this modulation has been described in terms of "gain field," in analogy to what is observed in two dimensions for oculomotor activities (Andersen et al., 1990; Bremmer et al., 1997b; Salinas and Thier, 2000). The present study shows that binocular eye position modulates the reach-distance tuning of neurons in area PE. We did not investigate the prevalence of a gain versus a shift effect because of the limited range of distances studied.

\section{Influence of hand position on reaching-related activity}

Information about hand position can be obtained from both visual and somatic information. It has been suggested that the control of movement distance is mainly based on proprioceptive signals and less on visual information, which is regarded as more important for the control of movement direction (Sainburg et al., 2003; Sarlegna et al., 2003; Bagesteiro et al., 2005). This is consistent with the evidence that these two parameters seem to be specified separately in the brain (Flanders et al., 1992; Lacquaniti et al., 1995; Georgopoulos, 2000). Area PE, contrary to areas MIP (medial intraparietal), V6a, or $7 \mathrm{~m}$, does not receive a direct visual input from extrastriate cortex (Johnson et al., 1996). In all these regions, retinal signals modulate reaching-related activity (Ferraina et al., 1997a,b; Batista et al., 1999; Battaglia-Mayer et al., 2000, 2001). The main source of somatic information to area PE arises from muscles, joints, and skin receptors and can be used to define hand position in intrinsic references frames. The importance of the SPL for the computation of the internal estimate of the initial hand position has been demonstrated also in humans by using transcranial magnetic stimulation (Vesia et al., 2008) and is in line with neuropsychological observations from parietal patients (Wolpert et al., 1998; Danckert et al., 2009). Our results, in agreement with the study by Lacquaniti et al. (1995), show that the initial hand position modulates the majority of the reachingrelated neurons in area PE, emphasizing the importance of hand position signals throughout the entire parieto-frontal system (Caminiti et al., 1990, 1991; Johnson et al., 1996). Other studies have shown arm position-related modulation of SPL neurons (Georgopoulos et al., 1984; Ferraina and Bianchi, 1994; Johnson et al., 1996; Battaglia-Mayer et al., 2000, 2001; Buneo et al., 2002; Averbeck et al., 2005; Fattori et al., 2005, 2009).

For reaching in two dimensions, it has been shown that limb position signals modulate the spatial tuning of reaching neurons in PRR (Snyder et al., 1997), 7m (Ferraina et al., 1997a,b), V6a (Battaglia-Mayer et al., 2000), and PEc (Ferraina et al., 2001), although the strength of this modulation versus that exerted by eye position differs across areas. Buneo et al. (2002) showed that limb position influence becomes stronger moving rostrally in the SPL, whereas Bhattacharyya et al. (2009) showed the importance of vergence in the posterior part of the SPL. These results are in agreement with the present ones and with those obtained by Johnson et al. (1996), Marconi et al. (2001), and Battaglia-Mayer et al. (2001), concerning the existence of gradients of reachrelated properties in the tangential domain of the SPL. An image of this gradient-like organization is reflected also in the properties of dorsocaudal premotor cortex, where the computations occurring in $\mathrm{PE}$ are addressed. In PMd, reach-neurons are influenced by hand-position (Caminiti et al., 1990, 1991; Burnod et al., 1992; Johnson et al., 1996) and by eye-position (Boussaoud et al., 1998; Pesaran et al., 2006) signals, although the influence of 
the former is greater than that of the latter (Cisek and Kalaska, 2002), as in area PE. These results provide experimental support to the conceptualization of the functional organization of the parieto-frontal system proposed by Caminiti et al. (2005), Burnod et al. (1999), and Battaglia-Mayer et al. (2003, 2006).

\section{Coordinate system for arm reaching in parietal area PE}

To successfully reach a target in space, the brain must build a correspondence between the representation of the target location and that of the movement end-point. Target location is encoded initially in eye- or head-centered coordinates (Andersen and Mountcastle, 1983; Andersen et al., 1985, 1990; Zipser and Andersen, 1988; Bremmer et al., 1997a,b) and must be progressively mapped in a body-centered system for an easy computation of the motor error (i.e., the vector of the desired hand movement).

By virtue of its position in the parieto-frontal system in general, and in the parietal gradient in particular, area PE is an advanced node in the conversion of different signals into limb coordinates. Our data show that neural modulation in area PE is mainly explained by the component in depth of the motor error. The influence of the motor error signal during a trial became more and more prevalent from visually to movement-related epochs. A shoulder-centered reference plane for reaching has been proposed to explain the modulation of neural activity during reaching to visual targets in depth in area PE (Lacquaniti et al., 1995). One important difference of our study is that monkeys reached to a memorized target and in total darkness. This condition could have increased the weight of hand proprioceptive signals, thus favoring in the process of a multiple-stage transformation (Flanders et al., 1992) and in the progressive match of reach-relevant information (Burnod et al., 1992, 1999) the emergence of a hand-centered representation for reaches (Gordon et al., 1994).

Our results support the idea that multiple coordinate transformation schemes coexist in the PPC, as a reflection of the multiplicity of task demands (Battaglia-Mayer et al., 2003). This view is supported by several modeling studies (Pouget and Sejnowski, 1997; Xing and Andersen, 2000). Pouget and Sejnowski (1997) have suggested that PPC's neurons combine multiple sources of information into a representation that allows a simultaneous readout in several frames of reference. Neurons in areas V6a, 7m, $\mathrm{PEc}$, and 7a combine different eye and hand signals in a spatially congruent manner (Battaglia-Mayer et al., 2000, 2001, 2005) within their global tuning field, a spatial frame suitable to provide a representation of reaching bound to task demands. Results from network models (Mascaro et al., 2003) further support this view.

To conclude, in the chain of operations believed to occur from target presentation to motor commands, target localization in depth is achieved in different areas by computing reaching distance in a reference frame based on the integration of binocular eye- and hand-related signals. Our results point to a role of area $\mathrm{PE}$, as the parietal area closest to the motor output mechanisms of the frontal lobe, in representing target location predominantly in hand-centered coordinates. Therefore, our results extend to reaching in depth the crucial role played by the SPL in the control of arm movement.

\section{References}

Andersen RA, Mountcastle VB (1983) The influence of the angle of gaze upon the excitability of the light-sensitive neurons of the posterior parietal cortex. J Neurosci 3:532-548.
Andersen RA, Essick GK, Siegel RM (1985) Encoding of spatial location by posterior parietal neurons. Science 230:456-458.

Andersen RA, Bracewell RM, Barash S, Gnadt JW, Fogassi L (1990) Eye position effects on visual, memory, and saccade-related activity in areas LIP and 7A of macaque. J Neurosci 10:1176-1196.

Andersen RA, Snyder LH, Bradley DC, Xing J (1997) Multimodal representation of space in the posterior parietal cortex and its use in planning movements. Annu Rev Neurosci 20:303-330.

Averbeck BB, Chafee MV, Crowe DA, Georgopoulos AP (2005) Parietal representation of hand velocity in a copy task. J Neurophysiol 93: $508-518$.

Bagesteiro LB, Sarlegna FR, Sainburg RL (2005) Differential influence of vision and proprioception on control of movement distance. Exp Brain Res 171:358-370.

Batista AP, Buneo CA, Snyder LH, Andersen RA (1999) Reach plans in eyecentered coordinates. Science 285:257-260.

Battaglia-Mayer A, Caminiti R (2002) Optic ataxia as result of the breakdown of the global tuning fields of parietal neurons. Brain 125:1-13.

Battaglia-Mayer A, Ferraina S, Mitsuda T, Marconi B, Genovesio A, Onorati P, Lacquaniti F, Caminiti R (2000) Early coding of reaching in the parietooccipital cortex. J Neurophysiol 83:2374-2391.

Battaglia-Mayer A, Ferraina S, Genovesio A, Marconi B, Squatrito S, Molinari M, Lacquaniti F, Caminiti R (2001) Eye-hand coordination during reaching. II. An analysis of the relationships between visuomanual signals in parietal cortex and parieto-frontal association projections. Cereb Cortex 11:528-544.

Battaglia-Mayer A, Caminiti R, Lacquaniti F, Zago M (2003) Multiple levels of representation of reaching in the parieto-frontal network. Cereb Cortex 13:1009-1022.

Battaglia-Mayer A, Mascaro M, Brunamonti E, Caminiti R (2005) The overrepresentation of contralateral space in parietal cortex: a positive image of directional motor components of neglect? Cereb Cortex 15:514-525.

Battaglia-Mayer A, Archambault PS, Caminiti R (2006) The cortical network for eye-hand coordination and its relevance to understanding motor disorders of parietal patients. Neuropsychologia 44:2607-2620.

Bhattacharyya R, Musallam S, Andersen RA (2009) Parietal reach region encodes reach depth using retinal disparity and vergence angle signals. J Neurophysiol 102:805-816.

Blangero A, Ota H, Delporte L, Revol P, Vindras P, Rode G, Boisson D, Vighetto A, Rossetti Y, Pisella L (2007) Optic ataxia is not only "optic": impaired spatial integration of proprioceptive information. Neuroimage 36 [Suppl 2]:T61-T68.

Blangero A, Gaveau V, Luaute J, Rode G, Salemme R, Guinard M, Boisson D, Rossetti Y, Pisella L (2008) A hand and a field effect in on-line motor control in unilateral optic ataxia. Cortex 44:560-568.

Boussaoud D, Jouffrais C, Bremmer F (1998) Eye position effects on the neuronal activity of dorsal premotor cortex in the macaque monkey. J Neurophysiol 80:1132-1150.

Brain WR (1941) Visual disorientation with special reference to lesions of the right hemisphere. Brain 64:224-272.

Bremmer F, Ilg UJ, Thiele A, Distler C, Hoffmann KP (1997a) Eye position effects in monkey cortex. I. Visual and pursuit-related activity in extrastriate areas MT and MST. J Neurophysiol 77:944-961.

Bremmer F, Distler C, Hoffmann KP (1997b) Eye position effects in monkey cortex. II. Pursuit- and fixation-related activity in posterior parietal areas LIP and 7A. J Neurophysiol 77:962-977.

Buneo CA, Jarvis MR, Batista AP, Andersen RA (2002) Direct visuomotor transformations for reaching. Nature 416:632-636.

Burnod Y, Otto I, Grandguillaume P, Ferraina S, Johnson PB, Caminiti R (1992) Visuomotor transformations underlying arm movements toward visual targets: a neural network model of cerebral cortical operations. J Neurosci 12:1435-1453.

Burnod Y, Baraduc P, Battaglia-Mayer A, Guigon E, Koechlin E, Ferraina S, Lacquaniti F, Caminiti R (1999) Parieto-frontal operations underlying arm reaching movement to visual targets: an integrated framework. Exp Brain Res 129:325-346.

Caggiano V, Fogassi L, Rizzolatti G, Thier P, Casile A (2009) Mirror neurons differentially encode the peripersonal and extrapersonal space of monkeys. Science 324:403-406.

Caminiti R, Johnson PB, Urbano A (1990) Making arm movements within different parts of space: dynamic aspects in the primate motor cortex. J Neurosci 10:2039-2058. 
Caminiti R, Johnson PB, Galli C, Ferraina S, Burnod Y (1991) Making arm movements within different parts of space: the premotor and motor cortical representation of a coordinate system for reaching to visual targets. J Neurosci 11:1182-1197.

Caminiti R, Ferraina S, Battaglia-Mayer A, Mascaro M, Burnod Y (2005) Parallel fronto-parietal circuits for sensorimotor transformation. In: Higher order motor disorders: from neuroanatomy and neurobiology to clinical neurology (Jeannerod M, Hallet M, Freund HJ, Leiguarda R, eds) pp 23-42. New York: Oxford UP.

Cisek P, Kalaska JF (2002) Modest gaze-related discharge modulation in monkey dorsal premotor cortex during a reaching task performed with free fixation. J Neurophysiol 88:1064-1072.

Cohen YE, Andersen RA (2002) A common reference frame for movement plans in the posterior parietal cortex. Nat Rev Neurosci 3:553-562.

Critchley M (1953) Tactile thought, with special reference to the blind. Brain 76:19-35.

Danckert J, Goldberg L, Broderick C (2009) Damage to superior parietal cortex impairs pointing in the sagittal plane. Exp Brain Res 195: 183-191.

Darian-Smith C, Darian-Smith I, Burman K, Ratcliffe N (1993) Ipsilateral cortical projections to area $3 \mathrm{a}, 3 \mathrm{~b}$, and area 4 in the macaque monkey. J Comp Neurol 335:200-213.

Dickey DA, Rawlings JO, Pantula SG (1998) Applied regression analysis. Berlin: Springer.

Dobbins AC, Jeo RM, Fiser J, Allman JM (1998) Distance modulation of neural activity in the visual cortex. Science 281:552-555.

Fattori P, Kutz DF, Breveglieri R, Marzocchi N, Galletti C (2005) Spatial tuning of reaching activity in the medial parieto-occipital cortex (area V6A) of macaque monkey. Eur J Neurosci 22:956-972.

Fattori P, Breveglieri R, Marzocchi N, Filippini D, Bosco A, Galletti C (2009) Hand orientation during reach-to-grasp movements modulates neuronal activity in the medial posterior parietal area V6A. J Neurosci 29: $1928-1936$

Ferraina S, Bianchi L (1994) Posterior parietal cortex: functional properties of neurons in area 5 during an instructed-delay reaching task within different parts of space. Exp Brain Res 99:175-178.

Ferraina S, Johnson PB, Garasto MR, Battaglia-Mayer A, Ercolani L, Bianchi L, Lacquaniti F, Caminiti R (1997a) Combination of hand and gaze signals during reaching: activity in parietal area $7 \mathrm{~m}$ in the monkey. J Neurophysiol 77:1034-1038.

Ferraina S, Garasto MR, Battaglia-Mayer A, Ferraresi P, Johnson PB, Lacquaniti F, Caminiti R (1997b) Visual control of hand-reaching movement: activity in parietal area 7m. Eur J Neurosci 9:1090-1095.

Ferraina S, Battaglia-Mayer A, Genovesio A, Marconi B, Onorati P, Caminiti R (2001) Early coding of visuomanual coordination during reaching in parietal area PEc. J Neurophysiol 85:462-465.

Flanders M, Helms-Tillery SI, Soechting JF (1992) Early stages in a sensorimotor transformation. Behav Brain Sci 15:309-362.

Foley JM (1980) Binocular distance perception. Psychol Rev 87:411-434.

Genovesio A, Ferraina S (2004) Integration of retinal disparity and fixation-distance related signals toward an egocentric coding of distance in the posterior parietal cortex of primates. J Neurophysiol 91:2670-2684.

Georgopoulos AP (2000) Neural aspects of cognitive motor control. Curr Opin Neurobiol 10:238-241.

Georgopoulos AP, Caminiti R, Kalaska JF (1984) Static spatial effects in motor cortex and area 5: quantitative relations in a two-dimensional space. Exp Brain Res 54:446-454.

Gnadt JW (1992) Area LIP: three dimensional space and visual to oculomotor transformation. Behav Brain Sci 15:745-746.

Gonzalez F, Perez R (1998) Modulation of cell responses to horizontal disparities by ocular vergence in the visual cortex of the awake Macaca mulatta monkey. Neurosci Lett 245:101-104.

Gordon J, Ghilardi MF, Ghez C (1994) Accuracy of planar reaching movements. I. Independence of direction and extent variability. Exp Brain Res 99:97-111.

Hasebe H, Oyamada H, Kinomura S, Kawashima R, Ouchi Y, Nobezawa S, Tsukada H, Yoshikawa E, Ukai K, Takada R, Takagi M, Abe H, Fukuda H, Bando T (1999) Human cortical areas activated in relation to vergence eye movements—a PET study. Neuroimage 10:200-208.
Holmes G, Horrax G (1919) Disturbances of spatial orientation and visual attention with loss of stereoscopic vision. Arch Neurol Psychiatry 1:385-407.

Johnson PB, Ferraina S, Bianchi L, Caminiti R (1996) Cortical networks for visual reaching: physiological and anatomical organization of frontal and parietal lobe arm regions. Cereb Cortex 6:102-119.

Jones EG, Coulter JD, Hendry SH (1978) Intracortical connectivity of architectonic fields in the somatic sensory, motor and parietal cortex of monkeys. J Comp Neurol 181:291-347.

Kalaska JF, Caminiti R, Georgopoulos AP (1983) Cortical mechanisms related to direction of two dimensional arm movements: relations in parietal area 5 and comparison with motor cortex. Exp Brain Res $51: 247-260$.

Kapoula Z, Isotalo E, Muri RM, Bucci MP, Rivaud-Pechoux S (2001) Effects of transcranial magnetic stimulation of the posterior parietal cortex on saccades and vergence. Neuroreport 12:4041-4046.

Khan AZ, Crawford JD, Blohm G, Urquizar C, Rossetti Y, Pisella L (2007) Influence of initial hand and target position on reach errors in optic ataxic and normal subjects. J Vis 7:1-16.

Lacquaniti F, Guigon E, Bianchi L, Ferraina S, Caminiti R (1995) Representing spatial information for limb movement: role of area 5 in the monkey. Cereb Cortex 5:391-409.

Marconi B, Genovesio A, Battaglia-Mayer A, Ferraina S, Squatrito S, Molinari M, Lacquaniti F, Caminiti R (2001) Eye-hand coordination during reaching. I. Anatomical relationships between parietal and frontal cortex. Cereb Cortex 11:513-527.

Mascaro M, Battaglia-Mayer A, Nasi L, Amit D, Caminiti R (2003) The eye and the hand: neural mechanisms and network models for oculomanual coordination. Cereb Cortex 13:1276-1286.

Myers RH (1986) Classical and modern regression with applications. Boston: Duxbury.

Oscarsson O, Rosén I (1963) Projection to cerebral cortex of large muscle-spindle afferents in the forelimb nerves of the cat. J Physiol 169:924-945.

Pandya DN, Seltzer B (1982) Intrinsic connections and architectonics of posterior parietal cortex in the rhesus monkey. J Comp Neurol 204:196-210.

Pesaran B, Nelson MJ, Andersen RA (2006) Dorsal premotor neurons encode the relative position of the hand, eye, and goal during reach planning. Neuron 51:125-134.

Pouget A, Sejnowski TJ (1994) A neural model of the cortical representation of egocentric distance. Cereb Cortex 4:314-329.

Pouget A, Sejnowski TJ (1997) A new view of hemineglect based on the response properties of parietal neurones. Philos Trans R Soc Lond B Biol Sci 352:1449-1459.

Raffi M, Ballabeni A, Maioli MG, Squatrito S (2008) Neuronal responses in macaque area PEc to saccades and eye position. Neuroscience 156: 413-424.

Rondot P, de Recondo J, Dumas JL (1977) Visuomotor ataxia. Brain 100:355-376.

Rosenbluth D, Allman JM (2002) The effect of gaze angle and fixation distance on the responses of neurons in V1, V2, and V4. Neuron 33:143-149.

Roy JP, Komatsu H, Wurtz RH (1992) Disparity sensitivity of neurons in monkey extrastriate area MST. J Neurosci 12:2478-2492.

Sainburg RL, Lateiner JE, Latash ML, Bagesteiro LB (2003) Effects of altering initial position on movement direction and extent. J Neurophysiol 89:401-415.

Sakata H, Shibutani H, Kawano K, Harrington TL (1985) Neural mechanisms of space vision in the parietal association cortex of the monkey. Vision Res 25:453-463.

Salinas E, Thier P (2000) Gain modulation: a major computational principle of the central nervous system. Neuron 27:15-21.

Sarlegna F, Blouin J, Bresciani JP, Bourdin C, Vercher JL, Gauthier GM (2003) Target and hand position information in the online control of goal-directed arm movements. Exp Brain Res 151:524-535.

Schlag-Rey M, Schlag J (1984) Visuomotor functions of central thalamus in monkey. I. Unit activity related to spontaneous eye movements. J Neurophysiol 51:1149-1174.

Schwartz AB, Kettner RE, Georgopoulos AP (1988) Primate motor cortex and free arm movements to visual targets in three-dimensional space. I. Relations between single cell discharge and direction of movement. J Neurosci 8:2913-2927. 
Snyder LH, Batista AP, Andersen RA (1997) Coding of intention in the posterior parietal cortex. Nature 386:167-170.

Taira M, Boline J, Smyrnis N, Georgopoulos AP, Ashe J (1996) On the relations between single cell activity in the motor cortex and the direction and magnitude of three-dimensional static isometric force. Exp Brain Res 109:367-376.

Tanaka M (2007) Spatiotemporal properties of eye position signals in the primate central thalamus. Cereb Cortex 17:1504-1515.

Trotter Y, Celebrini S, Stricanne B, Thorpe S, Imbert M (1996) Neural processing of stereopsis as a function of viewing distance in primate visual cortical area V1. J Neurophysiol 76:2872-2885.

Vesia M, Yan X, Henriques DY, Sergio LE, Crawford JD (2008) Transcranial magnetic stimulation over human dorsal-lateral posterior parietal cortex disrupts integration of hand position signals into the reach plan. J Neurophysiol 100:2005-2014.

Viguier A, Clement G, Trotter Y (2001) Distance perception within near visual space. Perception 30:115-124. von Hofsten C (1976) The role of convergence in visual space perception. Vision Res 16:193-198.

von Hofsten C (1977) Binocular convergence as a determinant of reaching behavior in infancy. Perception 6:139-144.

Wolpert DM, Goodbody SJ, Husain M (1998) Maintaining internal representations: the role of the human superior parietal lobe. Nat Neurosci 1:529-533.

Xing J, Andersen RA (2000) Models of the posterior parietal cortex which perform multimodal integration and represent space in several coordinate frames. J Cogn Neurosci 12:601-614.

Zhang M, Wang X, Goldberg ME (2008) Monkey primary somatosensory cortex has a proprioceptive representation of eye position. Prog Brain Res 171:37-45.

Zipser D, Andersen RA (1988) A back-propagation programmed network that simulates response properties of a subset of posterior parietal neurons. Nature 331:679-684. 